\title{
Verbal Fluency as a Rapid Screening Test for Cognitive Impairment in Early Parkinson's Disease
}

Teresa Torralva, Psy.D., Teresa Laffaye, Psy.D., Sandra Báez, Ph.D., Ezequiel Gleichgerrcht, M.D., Diana Bruno, Psy.D., Anabel Chade, M.D., Agustin Ibañez, Ph.D., Facundo Manes, M.D., Oscar Gershanik, M.D., María Roca, Ph.D.

The phonological verbal fluency test can act as a fast screening test to detect cognitive deficits in neurological conditions. In the present study, its utility in the detection of executive deficits in patients with early Parkinson's disease is demonstrated.

JNP in Advance (doi: 10.1176/appi.neuropsych.14060139)

Early detection and effective management of the nonmotor symptoms of Parkinson's disease (PD) is integral to the quality of life of both patients and caregivers. Even though cognitive impairment in the early stages of the disease may be subtle, the presence of early cognitive deficits is now well established, with executive function deficits being the most frequently reported. ${ }^{1-7}$

Even if an extensive evaluation of executive functions is critical to obtain an accurate description of a patient's neuropsychological profile, such evaluations are not always available to clinicians working with patients with PD. Thus, the existence of a brief screening tool, which is easy to administer and yet shows high sensitivity and specificity in the detection of executive deficits in patients with early PD would be of great value for clinicians working with patients with PD.

Faced with a verbal fluency test, subjects are asked to enumerate either words beginning with a given letter within a limited amount of time (phonological fluency) or words within a given category, such as animals (semantic fluency). These tasks are easy to administer, take no longer than 2 minutes and require no specific materials. The phonological version is thought to reflect more strongly on executive functioning, as it demands that participants list words using a rule that is not natural to the way words are organized in our brain. ${ }^{8}$ The phonological fluency test has been shown useful in the detection of cognitive deficits in pathologies with frontal involvement. ${ }^{9}$ Even if verbal fluency represents a well-known test of executive function with established age-based norms, its properties as screening tool in the detection of broader executive dysfunction in PD has not yet been established.

The objective of the present study was, therefore, to investigate the utility of the phonological verbal fluency test in the detection of executive deficits in patients with early PD.

\section{METHODS}

Permission for the study was initially obtained from the local research ethics committee, and all participants signed an informed consent prior to inclusion.

Thirty-two patients who met the United Kingdom Parkinson's Disease Society Brain Bank criteria, between Hoehn and Yahr stages I to II, ${ }^{10}$ were recruited for a broader ongoing study at the Institute of Cognitive Neurology (INECO). Twenty-two control subjects, matched by age, educational level, and premorbid intelligence were also included in the study. Control volunteers were obtained through word of mouth and were only included if they reported no history of neurological or psychiatric disorders. PD patients were grouped as either having (PDED N=15) on not having executive dysfunction (PDNED N=17) based on their performance in gold-standard executive tests (see below). It was considered that a patient had executive dysfunction if performance on at least one tests of the executive battery was more than 1.5 standard deviations under the mean of controls.

\section{Neuropsychological Assessment}

Verbal fluency task. All subjects were assessed with the standard Argentinean phonemic version of the phonological verbal fluency test, ${ }^{11}$ asking subjects to generate words beginning with the letter P in a 1-minute block. Score was the total number of correct words generated.

Standard executive battery. Subjects were also assessed with gold-standard executive tests usually used to assess executive functions in patients with PD. Such executive battery included the Trail Making Test Part B ${ }^{12}$ Digits Backward Test, ${ }^{13}$ and the Wisconsin Card Sorting Test. ${ }^{14}$ 
TABLE 1. Neuropsychological Performance PDED, PDNED, and Control Groups ${ }^{a}$

\begin{tabular}{|c|c|c|c|c|c|c|c|}
\hline \multirow{2}{*}{ Characteristic } & \multicolumn{2}{|c|}{ PDED } & \multicolumn{2}{|c|}{ PDNED } & \multicolumn{2}{|c|}{ Controls } & \multirow[b]{2}{*}{$\mathrm{p}$} \\
\hline & Mean & SD & Mean & SD & Mean & SD & \\
\hline Age (years) & 68.27 & 7.19 & 56.94 & 9.66 & 59.27 & 11.98 & 0.00 \\
\hline Years of formal education & 13.33 & 4.76 & 14.41 & 4.93 & 14.50 & 2.79 & 0.669 \\
\hline WAT & 35.47 & 5.04 & 38.18 & 3.32 & 38.68 & 2.93 & 0.036 \\
\hline ACE & 89.07 & 7.69 & 93.7 & 4.83 & 95.82 & 4.63 & 0.003 \\
\hline Digits Forward & 5.87 & 0.91 & 6.53 & 0.87 & 7.32 & 1.12 & 0.00 \\
\hline Digits Backward & 4.13 & 0.91 & 4.47 & 0.62 & 4.82 & 1.09 & 0.09 \\
\hline Verbal Fluency & 11.60 & 3.96 & 16.71 & 4.19 & 18.09 & 5.07 & 0.00 \\
\hline Trail Making $(\mathrm{A})$ & 67.33 & 47.39 & 38.76 & 12.54 & 34.86 & 15.12 & 0.00 \\
\hline Trail Making (B) & 183.27 & 122.36 & 77.00 & 20.49 & 79.86 & 40.44 & 0.00 \\
\hline WCST & 3.14 & 1.35 & 5.82 & 0.39 & 5.55 & 0.80 & 0.00 \\
\hline
\end{tabular}

${ }^{a}$ ACE =Addenbrooke's Cognitive Examination; WAT=Word Accentuation Test; $W C S T=$ Wisconsin Card Sorting Test.

Data analysis. The demographic and neuropsychological data were compared between the groups using analysis of variance (ANOVA) and Tukey honestly significant difference post hoc tests (when appropriate). To control for the influence of age on executive functions tasks, we applied analysis of covariance (ANCOVA) tests adjusted for age. The $\alpha$ value for all statistical tests was set at 0.05 .

To compare the usefulness of the IFS (INECO Frontal Screening) in detecting the executive dysfunction of patients with PD, we determined the sensitivity and specificity of this test to discriminate between 1 ) healthy controls and $\mathrm{PD}$ patients (PDED and PDNED), and 2) PDED and PDNED patients. This was done by means of a receiver operating characteristic curve, detecting the optimal cut-off scores. The area under the receiver operating characteristic curve was used as a measure of discriminatory accuracy

\section{RESULTS}

Demographic profile and total scores on tests of general cognitive status are summarized in Table 1. No significant difference was found for age $(t(52)=0.98$; $\mathrm{p}=0.33)$ years of formal education $(\mathrm{t}(29)=-0.52$; $\mathrm{p}=0.57)$ nor premorbid IQ $(\mathrm{t}(52)=-1.66 ; \mathrm{p}=0.10)$ between PD patients and controls. Significant differences were found for age between PDED patients and controls (F2, 51=5, 54; $\mathrm{p}=0.028)$ and between the former and PDNED patients $(\mathrm{F} 2,51=5,54 ; \mathrm{p}=0.007)$.

Significant differences between groups were found on the verbal fluency test (F (2, $50)=6.87, p<0.01, \eta^{2}=0.21$ ). Although controls showed a mean of $18.09(\mathrm{SD}=5.07)$, PDNED presented a mean of $16.71(\mathrm{SD}=4.19)$ and PDED a mean of $11.60(\mathrm{SD}=3.96)$ words in this test. A post hoc analysis (Tukey honestly significant difference, $\mathrm{MS}=20.51, \mathrm{df}=50$ ) revealed that controls performed significantly better than both PDED $(\mathrm{p}<0.01)$ and PDNED $(p<0.001)$. Moreover, both PD groups differed significantly between each other $(p<0.01)$, with PDNED outperforming PDED. As expected, significant differences between groups were also found on most gold-standard executive tests. In this regard, significant differences were found in in the Trail Making Test Part B $(\mathrm{F}(2,50)=6.60 ; \mathrm{p}<0.01$, $\eta^{2}=0.20$ ). A post hoc analysis (Tukey honestly significant difference, $\mathrm{MS}=4383.6, \mathrm{df}=50)$ showed that both controls $(p<0.01)$ and PDNED $(p<0.01)$ performed better than PDED, even if a significant effect of age $(p<0.01)$ was observed on Trail Making Test Part B performance. Significant differences between groups were also found on the WCST (Wisconsin Card Sorting Test $)\left(F(2,50)=29.46, p<0.01, \eta^{2}=0.50\right)$. A post hoc analysis (Tukey honestly significant difference, $\mathrm{MS}=0.76, \mathrm{df}=50)$ revealed that PDNED $(\mathrm{p}<0.01)$ and controls $(p<0.01)$ exhibited a higher performance than PDED. Unexpectedly, no significant differences were found between groups on the Digits Backward Test $(\mathrm{F}(2,50)=2.50, \mathrm{p}=0.92$, $\left.\eta^{2}=0.09\right)$. This lack of significant differences can be related to

FIGURE 1. Verbal Fluency Receiver Operating Characteristic Curve ${ }^{a}$ PD (ED) vs. PD (NED) vs. CTR
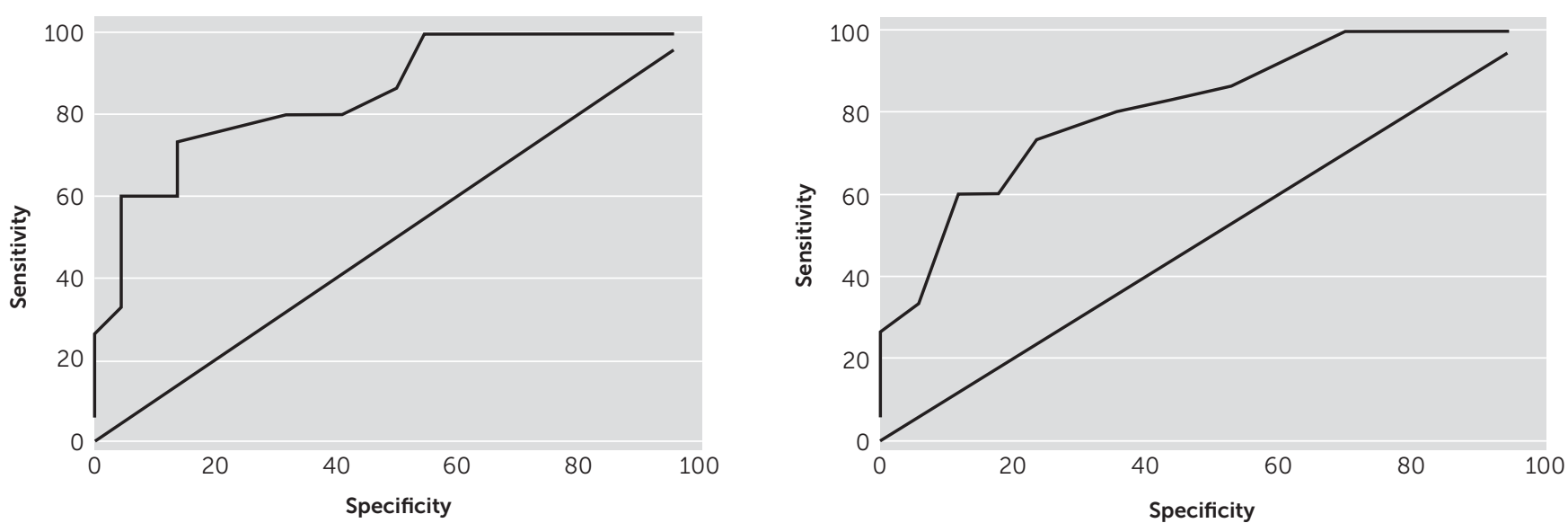

${ }^{a}$ [A] Receiver operating characteristic curve analysis between PDED, PDNED, and control groups. [B] Verbal fluency receiver operating characteristic curve analysis between PDED and PDNED. 
the fact that Digit Backward Test relies on verbal instead of visual working memory, the latter of which is most commonly decreased in early PD patients. ${ }^{15}$

Significant correlations were found between verbal fluency score, and the total number of categories abstracted on the WCST $(\mathrm{r}=0.52 ; \mathrm{p}<0.001)$, and time to complete the TMT-B $(\mathrm{r}=-0.82 ; \mathrm{p}<0.001)$. No significant correlations were found between the verbal fluency score and the Digits Backward Test $(\mathrm{r}=0.23 ; \mathrm{p}<0.01)$

A receiver operating characteristic curve analysis on the verbal fluency total score comparing healthy controls and PD patients (PDED and PDNED) generated a cut-off score of 14 points with sensitivity of $73.3 \%$ and specificity of $82.1 \%$ (Figure 1A).

With a verbal fluency score of $14,73.3 \%$ of the PDED patients, $76.4 \%$ of the PDNED patients, and $86.3 \%$ of the controls were correctly classified. Area under the receiver operating characteristic curve was 0.85 [confidence interval (CI) 0.72-0.97; $\mathrm{p}<0.01]$. Furthermore, when patients were divided into PDED and PDNED, a receiver operating characteristic curve analysis comparing both groups generated a cut-off score of 14 points, with a sensitivity of $73.3 \%$ and a specificity of $76.4 \%$ and an area under the curve of 0.81 (CI 0.66-0.96; $\mathrm{p}<0.01$ ) (Figure 1B).

\section{DISCUSSION}

In the present study, we showed that the phonological verbal fluency task may be a good screening test for the detection of executive deficits in PD. Our findings show adequate concurrent validity, as revealed by the significant correlations that the test showed with executive tasks, and adequate discriminant validity, as evidenced by the test's capability to significantly differentiate PDED patients from healthy controls and from PDNED patients. All the between-group differences remained significant even after controlling for the effect of age save an effect of age observed on the Trail Making Test B performance. This is not unexpected, as several studies $^{16-18}$ have shown that among adults processing speed is positively correlated with age.

Using a cut-off score of 14 points, the Verbal Fluency Task showed a sensitivity of $73.3 \%$ and a specificity of $76.4 \%$ in the detection of executive deficits in PD. These results suggest that this brief test is able to correctly classify the majority of PD patients with executive dysfunction and those without it.

Our results have important implications for clinicians working with patients with PD who cannot always access more extensive neuropsychological evaluations. The present investigation indicates that the phonological verbal fluency task can act as a sensitive and specific screening tool in the detection of executive deficits in this pathology. Even if an extensive evaluation of executive functions might be critical to obtain an accurate description of a patient's neuropsychological profile, this 2-minute screening tool, combined with an adequate interview regarding the impact of cognitive dysfunction in patients' lives, can be useful in the detection of patients who may benefit from more extensive cognitive evaluations and cognitively targeted interventions.

Although no single test can replace the value of a complete and focused history-taking or that of a comprehensive neuropsychological evaluation, the present study indicates that phonological verbal fluency can act as a reliable, brief, and easy-to administer tool for the screening of executive dysfunction in PD. In this regard, the phonological verbal fluency test could act as a simple tool to be used in everyday clinical practice to detect those patients with PD who will benefit from a more comprehensive neuropsychological battery, including a thorough cognitive history-taking and further test of executive and social functioning.

\section{AUTHOR AND ARTICLE INFORMATION}

From the Institute of Cognitive Neurology (INECO), Buenos Aires, Argentina (TT, TL, SB, EG, DB, AC, Al, FM, OG, MR); Favaloro University, Buenos Aires, Argentina (TT, AC, Al, FM, OG, MR); UDP-INECO Foundation Core on Neuroscience (UIFCoN), Diego Portales University, Santiago, Chile (TT, SB, Al, MR); the National Scientific and Technical Research Council (CONICET), Buenos Aires, Argentina (Al, MR); Universidad Autónoma del Caribe, Barranquilla, Colombia (Al); and the Centre of Excellence in Cognition and its Disorders, Australian Research Council (ACR), Sydney, Australia (AI).

Send correspondence to Dr. Roca; e-mail: mroca@ineco.org.ar.

Supported by grants from CONICET, CONICYT/FONDECYT Regular (1130920), FONCyT-PICT 2012-0412, FONCYT-PICT 2012-1309, and the INECO Foundation.

The authors report no financial relationships with commercial interests. Received June 24, 2014; revised Sept. 10, 2014; accepted Sept. 11, 2014.

\section{REFERENCES}

1. Foltynie T, Brayne CE, Robbins TW, et al: The cognitive ability of an incident cohort of Parkinson's patients in the UK. The CamPaIGN study. Brain 2004; 127:550-560

2. Muslimovic D, Post B, Speelman JD, et al: Cognitive profile of patients with newly diagnosed Parkinson disease. Neurology 2005; 65:1239-1245

3. Lewis SJ, Foltynie T, Blackwell AD, et al: Heterogeneity of Parkinson's disease in the early clinical stages using a data driven approach. $\mathrm{J}$ Neurol Neurosurg Psychiatry 2005; 76:343-348

4. Williams-Gray $\mathrm{CH}$, Foltynie T, Brayne $\mathrm{CE}$, et al: Evolution of cognitive dysfunction in an incident Parkinson's disease cohort. Brain 2007; 130:1787-1798

5. Dirnberger G, Jahanshahi M: Executive dysfunction in Parkinson's disease: a review. J Neuropsychol 2013; 7:193-224

6. Engeln M: Throwing some light on executive function in Parkinson's disease. Mov Disord 2013; 28:1052

7. Kudlicka A, Clare L, Hindle JV: Pattern of executive impairment in mild to moderate Parkinson's disease. Dement Geriatr Cogn Disord 2013; 36:50-66

8. Lezak MD: Neuropsychological Assessment. New York, Oxford University Press, 1995

9. Connick P, Kolappan M, Bak TH, et al: Verbal fluency as a rapid screening test for cognitive impairment in progressive multiple sclerosis. J Neurol Neurosurg Psychiatry 2012; 83:346-347

10. Hoehn MM, Yahr MD. Parkinsonism: onset, progression, and mortality. Neurology 1967; 17:427-42

11. Butman J, Allegri RF, Harris P, et al: Fluencia verbal en español. Datos normativos en Argentina. Medicina (B Aires) 2000; 60:561-564

12. Partington JE, Leiter RG: Partington's Pathway Test. Psychological Service Center Bulletin 1949; 1:9-20 
13. Wechsler D: WAIS - III. Escala de inteligencia para adultos de Wechsler. Tercera edición. Buenos Aires, Paidós, 2002

14. Nelson HE: A modified card sorting test sensitive to frontal lobe defects. Cortex 1976; 12:313-324

15. Owen AM, Iddon JL, Hodges JR, et al: Spatial and non-spatial working memory at different stages of Parkinson's disease. Neuropsychologia 1997; 35:519-532

16. Tamayo F, Casals-Coll M, Sánchez-Benavides G, et al: [Spanish normative studies in a young adult population (NEURONORMA young adults Project): norms for the verbal span, visuospatial span, Letter-Number Sequencing, Trail Making Test and Symbol Digit Modalities Test]. Neurologia 2012; 27:319-329

17. Riccio CA, Blakely A, Yoon M, et al: Two-factor structure of the Comprehensive Trail-Making Test in adults. Appl Neuropsychol Adult 2013; 20:155-158

18. LaRoche DP, Greenleaf BL, Croce RV, et al: Interaction of age, cognitive function, and gait performance in 50-80-year-olds. Age (Dordr) 2014; 36:9693 